\title{
Versatile monochromator module for XHR SEM.
}

\author{
A. Henstra *, J. Chmelik **, T. Dingle ( $\dagger)$, A. Mangnus *, G. van Veen * \\ * FEI Company, PO Box 80066, 5600 KA Eindhoven, The Netherlands. \\ ** FEI Czech Republic s.r.o., Podnikatelská 6, 61200 Brno, Czech Republic.
}

Low voltage SEM image resolution is predominantly limited by chromatic aberration. In order to strongly reduce this effect, FEI has incorporated a monochromator in its latest XHR SEM system (called the Magellan). This enables the reduction of energy spread to $0.15 \mathrm{eV}$ FWHM. To illustrate the potential of a monochromator for low voltage SEM, figure 1 shows the probe size as a function of energy spread, for accelerating voltages $U$ $=0.5$ and $1 \mathrm{kV}$. Sub-nm resolution Magellan images at low voltage have revealed unprecedented information, such as fine surface details and nanoparticle distributions, without any sample preparation [1].

An electrostatic Schottky-FEG module has been developed that can provide a monochromatized beam for XHR imaging, and which can also provide large probe currents for analysis. The three modes of operation of this module are shown in figure 2 . The extractor contains a first aperture plane with two apertures, defining an axial and offaxial beam. An electrode below the extractor serves as gun lens. This electrode is segmented, so it can also be used for deflection and stigmation. The second aperture plane has an axial aperture and a small, off-axial slit [2]. The latter is used to monochromatize the off-axial beam. A deflector below the second aperture plane is used to direct either the axial or off-axial beam into the column, where a final beam limiting aperture is used to determine the probe current. Unwanted electrons are blocked.

In the first mode of operation, the axial beam is used with gun lens off. The axial aperture in the second aperture plane limits the beam current. In the second mode of operation, the gun lens is turned on to deliver more beam current, which can go up to 22 nA. The third mode (called UC mode) uses the off-axial monochromatized beam. Voltages on the gun lens segments are used to position, focus and stigmate the off-axial beam on the energy selecting slit. The beam is dispersed due to the off-axial traversal through the strong gun lens. The spherical aberration of the gun lens results in coma in the off-axial beam. However, the effect of this coma on the energy spread is negligible for useful probe currents, i.e. $<100 \mathrm{pA}$. For larger probe currents, the loss of brightness becomes more important than the reduction in energy spread.

An example of a high resolution SE image at $1 \mathrm{kV}$ on a gold on carbon sample is displayed in figure 3. Sub-nm edge sharpness (25-75\%) has been achieved in such an image.

\section{References:}

[1] R. Young et al., Microscopy Today, jul. aug. issue (2008) 24.

[2] H.W. Mook, P. Kruit, Ultramicroscopy 81(3) (2000) 129.

[3] The authors gratefully acknowledge the support of the Smart Mix Programme of the Netherlands Ministry of Economic Affairs and the Netherlands Ministry of Education, Culture and Science. 


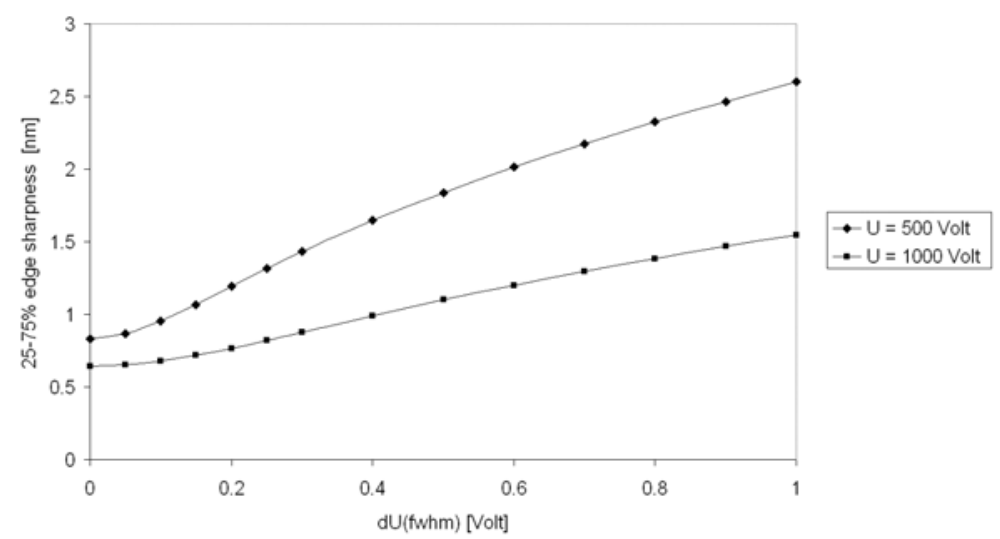

Figure 1. Theoretical probe size as a function of energy spread, for Magellan XHR SEM at working distance $=1 \mathrm{~mm}$, in the limit of low probe current, and at optimal opening angle. Typical energy spread of a Schottky-FEG $=0.7$ Volt FWHM.

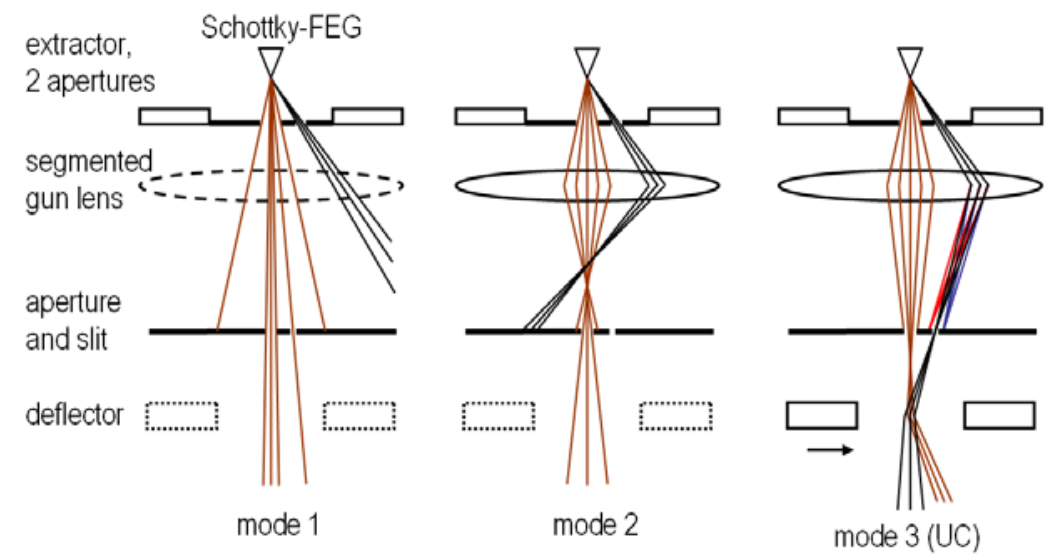

Figure 2. The three modes of the XHR gun module. From left to right: Standard on axis mode, high current mode and the UC mode.

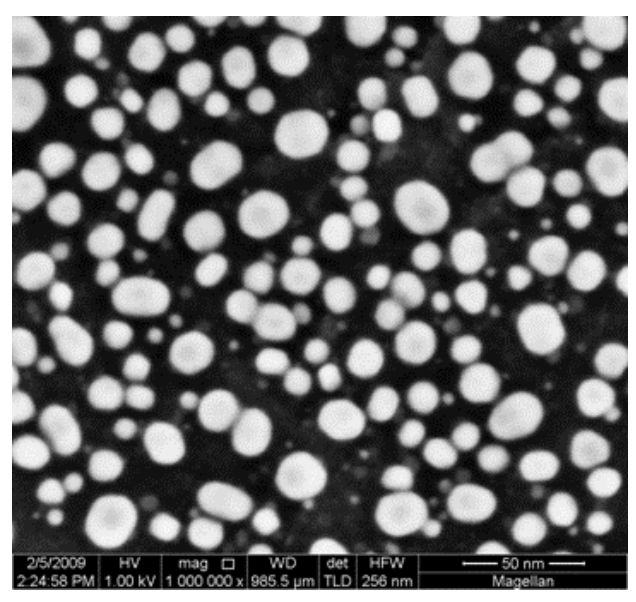

Figure 3. Magellan image of a gold on carbon sample showing XHR image resolution performance in UC mode. Beam deceleration is not applied, i.e. the stage is not negatively biased. 